\title{
Factores de personalidad y actitud hacia los residuos sólidos en estudiantes de una universidad particular de Lima - Perú.
}

Personality factors and attitude towards solid waste in students from a private university in Lima - Perú.

\author{
Rebeca Machicao Fonseca ${ }^{1}$
}

\section{RESUMEN}

La presente investigación examina la relación entre los cinco grandes factores de la personalidad y la actitud hacia los residuos sólidos. El diseño de la investigación es transaccional correlacional y la muestra la constituyeron 308 estudiantes universitarios procedentes de tres facultades de una universidad particular de Lima, Perú. Los instrumentos utilizados fueron el NEO FFI de Costa \&McCrae y la Escala de actitud hacia los residuos urbanos de Fernández y cols. Los resultados revelan que la actitud positiva hacia los residuos sólidos está relacionada a niveles altos en la apertura a la experiencia, responsabilidad, extraversión y agradabilidad, y a un nivel bajo de neuroticismo. Además se analiza la relación considerando el papel del género, período de desarrollo humano, ciclo académico, carrera y área de residencia.

PALABRAS CLAVE: Actitud, personalidad, residuos sólidos, modelo de los cinco factores

\section{SUMMARY}

The current research examines the relation among the big five factors of personality and attitude towards solid waste. The design of the research is transactional-correlational and the sample is composed by 308 university students, from three departments of a private university in Lima, Peru. The instruments used were the NEO FFI (Costa \& McCrae) and the Attitude towards urban waste scale (Fernández et.al.). The results reveal that positive attitude towards solid waste is related to high levels to the openness to experience, conscientiousness, extraversion and agreeableness and to low levels of neuroticism. Furthermore, the relationship is analyzed by considering the role of gender, human development period, academic term, career and area of residence.

KEYWORDS: Attitude, personality, solid waste, five-factor model. 


\section{INTRODUCCIÓN}

Durante las últimas décadas el impacto de la actividad humana sobre el medio ambiente y los consecuentes sucesos ambientales que terminan afectando a los sistemas humanos vienen recibiendo una creciente atención. Desde el interior de la psicología es la psicología ambiental la que trata contribuir en resolver los problemas medioambientales a través de soluciones comportamentales. Se han realizado, se han realizado gran número de investigaciones respecto a la influencia que ejercen los factores psicológicos sobre las actitudes y comportamientos tanto de protección, como de degradación del medio ambiente. Estos estudios se han enfocado principalmente en el papel de los valores, las normas y las creencias como predictores de la actitud hacia el ambiente.

Recientemente, las investigaciones han comenzado a examinar la personalidad como la característica que influye en las actitudes y comportamientos ambientales en diferentes situaciones. Costa \& McCrae (1994) definen a la personalidad como el conjunto de cinco grandes rasgos: neuroticismo, extraversión, apertura, agradabilidad y responsabilidad. Estos autores establecen un modelo de los cinco factores de personalidad que están presentes en todas las personas y que han demostrado ser transculturalmente válidos.

En base al modelo de los cinco factores, Hirsh \& Dolderman condujeron en el 2007 una investigación que relacionó los factores de personalidad con los valores de consumismo y ambientalismo. Estos autores reportaron que mientras el consumismo se encontró negativamente relacionado al factor agradabilidad, el ambientalismo se relacionó positivamente con agradabilidad y apertura a la experiencia. Así, los resultados de esta investigación apuntan a que los individuos con rasgos más empáticos y menos centrados en sí mismos, tienen una mayor probabilidad de desarrollar una conexión personal con la naturaleza.

En el 2010, Hirsh examinó la relación entre las características de la personalidad y la preocupación ambiental. Los resultados de su investigación señalan que mayores niveles de preocupación ambiental se encuentran relacionados a altos niveles de agradabilidad y de apertura a la experiencia, y con menores relaciones positivas con los factores de neuroticismo y responsabilidad. Respecto a agradabilidad, Hirsh asocia este factor a niveles elevados de empatía, que se vinculan a la motivación proambiental. Por su parte, el factor apertura a la experiencia es asociado con una perspectiva más amplia del lugar de la humanidad en la ecología general y el poseer una mayor apreciación estética de la belleza natural.

Bajo la línea de estas investigaciones, el presente estudio establece como objetivo central determinar la relación entre los cinco factores de personalidad y la actitud hacia los residuos sólidos en estudiantes universitarios, estableciendo además los siguientes objetivos específicos:

1. Determinar la relación entre los cinco factores de personalidad y la actitud hacia los residuos sólidos, según el género.

2. Determinar la relación entre los cinco factores de personalidad y la actitud hacia los residuos sólidos, según el periodo de desarrollo humano.

3. Determinar la relación entre los cinco factores de personalidad y la actitud hacia los residuos sólidos, según el ciclo académico.

4. Determinar la relación entre los cinco factores de personalidad y la actitud hacia los residuos sólidos, según la carrera.

5. Determinar la relación entre los cinco factores de personalidad y la actitud hacia los residuos sólidos, según el área de residencia.

6. Determinar los niveles de neuroticismo, extraversión, apertura a la experiencia, agradabilidad y responsabilidad en el grupo de estudio.

7. Determinar la actitud hacia los residuos sólidos en el grupo de estudio.

\section{MATERIAL Y MÉTODOS}

La investigación es no experimental. El diseño empleado fue el transaccional correlacional.

La muestra se seleccionó de forma no probabilística y estuvo conformada por 308 estudiantes de una universidad particular de Lima, Perú. La muestra estuvo constituida en un $79 \%$ por mujeres y en un $21 \%$ por varones. El $88 \%$ de la muestra presentó edades de entre 16 a 19 años y el $12 \%$, entre 20 a 25 años. El $51 \%$ se encontraba inscrito en el tercer ciclo y el $49 \%$, en el primer ciclo. El $42,9 \%$ de la muestra pertenecían a la carrera $\mathrm{N}^{\circ} 3$, el $34,4 \%$ a la carrera $\mathrm{N}^{\circ} 2$ y el $22,7 \%$ a la carrera $\mathrm{N}^{\circ} 1$. Asimismo, el $44,5 \%$ de la muestra residían en los distritos de Lima Residencial y un $28.6 \%$ en distritos de Lima Norte.

La evaluación de los cinco factores de personalidad se realizó con el NEO FII (Inventario NEO reducido 
de cinco factores) de Costa \& McCrae. El NEO FFI consta de 60 ítems y los participantes responden a través de cinco opciones: Totalmente en desacuerdo (A), en desacuerdo (B), neutral (C), de acuerdo (D) y totalmente de acuerdo (E). El estudio psicométrico del inventario fue realizado por Ramírez Ore (2007), concluyéndose que el inventario cumple con los requisitos fundamentales para su aplicación en el ámbito nacional.

La actitud hacia los residuos sólidos fue evaluada con la escala de actitud hacia los residuos urbanos desarrollada por Fernández, Hueto, Rodríguez y Marcén (2003). La escala está formada por 25 ítems, en formato Likert con cinco opciones de respuesta: Muy en desacuerdo, desacuerdo, neutral, de acuerdo y muy de acuerdo. Para su aplicación en el ámbito nacional, se realizó un estudio de sus propiedades psicométricas encontrándose niveles óptimos.

\section{RESULTADOS}

Con respecto al objetivo central, determinar la relación entre los cinco factores de personalidad y la actitud hacia los residuos sólidos. Los resultados descritos en la Tabla 1 muestran que los cinco factores de personalidad correlacionan con la actitud. Se demuestra, de tal forma, que la actitud positiva hacia los residuos sólidos está relacionada a mayores niveles de apertura a la experiencia, responsabilidad, extraversión y agradabilidad, así como a un bajo nivel de neuroticismo.

En relación al objetivo específico $\mathrm{N}^{\circ} 1$, determinar la relación entre los cinco factores de personalidad y la actitud hacia los residuos sólidos según el género, los resultados que se observan en la Tabla 2 , se reporta que la apertura a la experiencia se mantiene como el predictor más significativo de la actitud hacia los residuos sólidos en ambos géneros.

Tabla 1. Correlaciones entre los cinco factores de personalidad y la actitud hacia los residuos sólidos.

\begin{tabular}{ccccccc}
\hline & & $\mathrm{N}$ & $\mathrm{E}$ & $\mathrm{O}$ & $\mathrm{A}$ & $\mathrm{C}$ \\
\hline & Coeficiente de correlación de Pearson & $-0,122$ & 0,17 & 0,315 & 0,149 & 0,212 \\
Actitud & $\mathrm{N}$ & 308 & 308 & 308 & 308 & 308 \\
\hline
\end{tabular}

Tabla 2. Relación entre personalidad y actitud hacia los residuos sólidos, según género.

\begin{tabular}{|c|c|c|c|c|c|c|}
\hline & & $\mathrm{N}$ & $\mathrm{E}$ & $\mathrm{O}$ & A & $\mathrm{C}$ \\
\hline \multirow[t]{2}{*}{$\begin{array}{l}\text { Actitud } \\
\text { Hombres }\end{array}$} & $\begin{array}{c}\text { Coeficiente de correlación de } \\
\text { Pearson }\end{array}$ & $-0,255$ & 0,248 & 0,344 & $-0,044$ & 0,192 \\
\hline & $\mathrm{N}$ & 65 & 65 & 65 & 65 & 65 \\
\hline \multirow[t]{2}{*}{$\begin{array}{l}\text { Actitud } \\
\text { Mujeres }\end{array}$} & $\begin{array}{c}\text { Coeficiente de correlación de } \\
\text { Pearson }\end{array}$ & $-0,083$ & 0,130 & 0,307 & 0,198 & 0,220 \\
\hline & $\mathrm{N}$ & 243 & 243 & 243 & 243 & 243 \\
\hline
\end{tabular}

Tabla 3. Relación entre personalidad y actitud hacia los residuos sólidos, según período de desarrollo humano.

\begin{tabular}{ccccccc}
\hline & $\mathrm{N}$ & $\mathrm{E}$ & $\mathrm{O}$ & $\mathrm{A}$ & $\mathrm{C}$ \\
\hline $\begin{array}{c}\text { Actitud } \\
\text { Adolescencia }\end{array}$ & $\begin{array}{c}\text { Coeficiente de correlación } \\
\text { de Pearson }\end{array}$ & $-0,124$ & 0,197 & 0,345 & 0,166 & 0,236 \\
$\mathrm{~N}$ & 272 & 272 & 272 & 272 & 272 \\
$\begin{array}{c}\text { Actitud Adultez } \\
\text { Temprana }\end{array}$ & $\begin{array}{c}\text { Coeficiente de correlación } \\
\text { de Pearson }\end{array}$ & $-0,105$ & 0,118 & 0,128 & 0,108 & 0,111 \\
& $\mathrm{~N}$ & 36 & 36 & 36 & 36 & 36 \\
\hline
\end{tabular}


Tabla 4. Relación entre personalidad y actitud hacia los residuos sólidos, según ciclo académico.

\begin{tabular}{|c|c|c|c|c|c|c|}
\hline & & $\mathrm{N}$ & $E$ & $\mathrm{O}$ & A & $\mathrm{C}$ \\
\hline \multirow[t]{2}{*}{$\begin{array}{c}\text { Actitud } \\
\text { Primer ciclo }\end{array}$} & $\begin{array}{c}\text { Coeficiente de correlación } \\
\text { de Pearson }\end{array}$ & $-0,203$ & 0,233 & 0,357 & 0,155 & 0,342 \\
\hline & $\mathrm{N}$ & 151 & 151 & 151 & 151 & 151 \\
\hline \multirow[t]{2}{*}{$\begin{array}{l}\text { Actitud } \\
\text { Tercer ciclo }\end{array}$} & $\begin{array}{c}\text { Coeficiente de correlación } \\
\text { de Pearson }\end{array}$ & $-0,051$ & 0,099 & 0,270 & 0,135 & 0,083 \\
\hline & $\mathrm{N}$ & 157 & 157 & 157 & 157 & 157 \\
\hline
\end{tabular}

Tabla 5. Relación entre personalidad y actitud hacia los residuos sólidos, según carrera.

\begin{tabular}{ccccccc}
\hline & & $\mathrm{N}$ & $\mathrm{E}$ & $\mathrm{O}$ & $\mathrm{A}$ & $\mathrm{C}$ \\
\hline Actitud & $\begin{array}{c}\text { Coeficiente de } \\
\text { Carrera N }\end{array}$ & -0.137 & 0.144 & 0.131 & 0.246 & 0.290 \\
& $\begin{array}{c}\text { correlación de Pearson } \\
\mathrm{N}\end{array}$ & 70 & 70 & 70 & 70 & 70 \\
$\begin{array}{c}\text { Actitud } \\
\text { Carrera N } 2\end{array}$ & $\begin{array}{c}\text { Coeficiente de } \\
\text { correlación de Pearson }\end{array}$ & -0.135 & 0.014 & 0.335 & -0.046 & 0.011 \\
Actitud & $\begin{array}{c}\mathrm{N} \\
\text { Carrera N } 3\end{array}$ & 106 & 106 & 106 & 106 & 106 \\
& correlación de Pearson & -0.153 & 0.306 & 0.372 & 0.211 & 0.324 \\
\hline
\end{tabular}

Tabla 6. Relación entre personalidad y actitud hacia los residuos sólidos, según área de residencia.

\begin{tabular}{|c|c|c|c|c|c|c|}
\hline & & $\mathrm{N}$ & E & $\mathrm{O}$ & A & $\mathrm{C}$ \\
\hline \multirow[t]{2}{*}{$\begin{array}{c}\text { Actitud } \\
\text { Cercado de Lima }\end{array}$} & $\begin{array}{l}\text { Coeficiente de correlación } \\
\text { de Pearson }\end{array}$ & -0.110 & 0.113 & 0.188 & 0.047 & 0.438 \\
\hline & $\mathrm{N}$ & 29 & 29 & 29 & 29 & 29 \\
\hline \multirow[t]{2}{*}{$\begin{array}{c}\text { Actitud } \\
\text { Lima Residencial }\end{array}$} & $\begin{array}{l}\text { Coeficiente de correlación } \\
\text { de Pearson }\end{array}$ & -0.143 & 0.167 & 0.346 & 0.146 & 0.222 \\
\hline & $\mathrm{N}$ & 137 & 137 & 137 & 137 & 137 \\
\hline \multirow[t]{2}{*}{$\begin{array}{l}\text { Actitud } \\
\text { Lima Este }\end{array}$} & $\begin{array}{l}\text { Coeficiente de correlación } \\
\text { de Pearson }\end{array}$ & 0.174 & 0.173 & 0.587 & 0.286 & 0.253 \\
\hline & $\mathrm{N}$ & 23 & 23 & 23 & 23 & 23 \\
\hline \multirow{2}{*}{$\begin{array}{c}\text { Actitud } \\
\text { Lima Norte }\end{array}$} & $\begin{array}{l}\text { Coeficiente de correlación } \\
\text { de Pearson }\end{array}$ & -0.179 & 0.300 & 0.245 & 0.233 & 0.134 \\
\hline & $\mathrm{N}$ & 88 & 88 & 88 & 88 & 88 \\
\hline \multirow{2}{*}{$\begin{array}{l}\text { Actitud } \\
\text { Lima Sur }\end{array}$} & $\begin{array}{l}\text { Coeficiente de correlación } \\
\text { de Pearson }\end{array}$ & -0.205 & 0.047 & 0.424 & 0.043 & 0.284 \\
\hline & $\mathrm{N}$ & 18 & 18 & 18 & 18 & 18 \\
\hline \multirow{2}{*}{$\begin{array}{l}\text { Actitud } \\
\text { Callao }\end{array}$} & $\begin{array}{l}\text { Coeficiente de correlación } \\
\text { de Pearson }\end{array}$ & 0.290 & -0.256 & 0.151 & -0.218 & 0.099 \\
\hline & $\mathrm{N}$ & 13 & 13 & 13 & 13 & 13 \\
\hline
\end{tabular}


Sobre el objetivo específico $\mathrm{N}^{\circ} 2$, determinar la relación entre los cinco factores de personalidad y la actitud hacia los residuos sólidos según el período de desarrollo humano. se observa en la Tabla 3 que los cinco factores de personalidad influyen en la actitud de los individuos que se encuentran en el período de la adolescencia y la adultez temprana.

Respecto al objetivo específico $\mathrm{N}^{\circ} 3$, determinar la relación entre los cinco factores de personalidad y la actitud hacia los residuos sólidos según el ciclo académico, los resultados expuestos en la Tabla 4 indican que los rasgos de personalidad en los estudiantes del primer ciclo predicen en mayor nivel la actitud hacia los residuos sólidos, y cobran relevancia los factores de apertura y responsabilidad. Sin embargo, en los estudiantes del tercer ciclo solo apertura y agradabilidad explican la actitud positiva hacia los residuos sólidos.

En cuanto al objetivo específico $\mathrm{N}^{\circ} 4$, determinar la relación entre los cinco factores de personalidad y la actitud hacia los residuos sólidos según la carrera, se encontró que la agradabilidad y la responsabilidad son los factores más significativos en la predicción de la actitud en los estudiantes de la carrera número uno. En la carrera número dos, y en la carrera número tres, emergen la extroversión, apertura y responsabilidad (Ver Tabla 5).

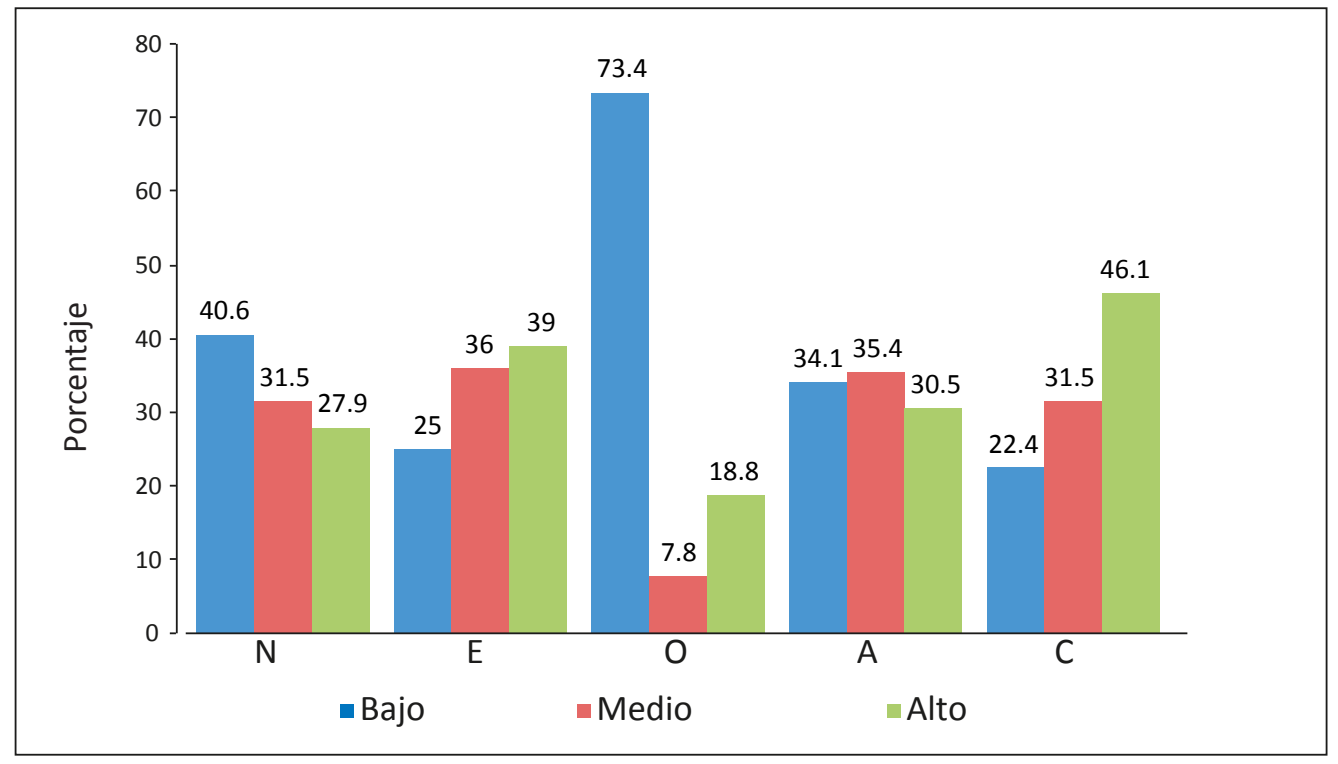

Figura 1. Distribución de resultados de cinco factores de personalidad.

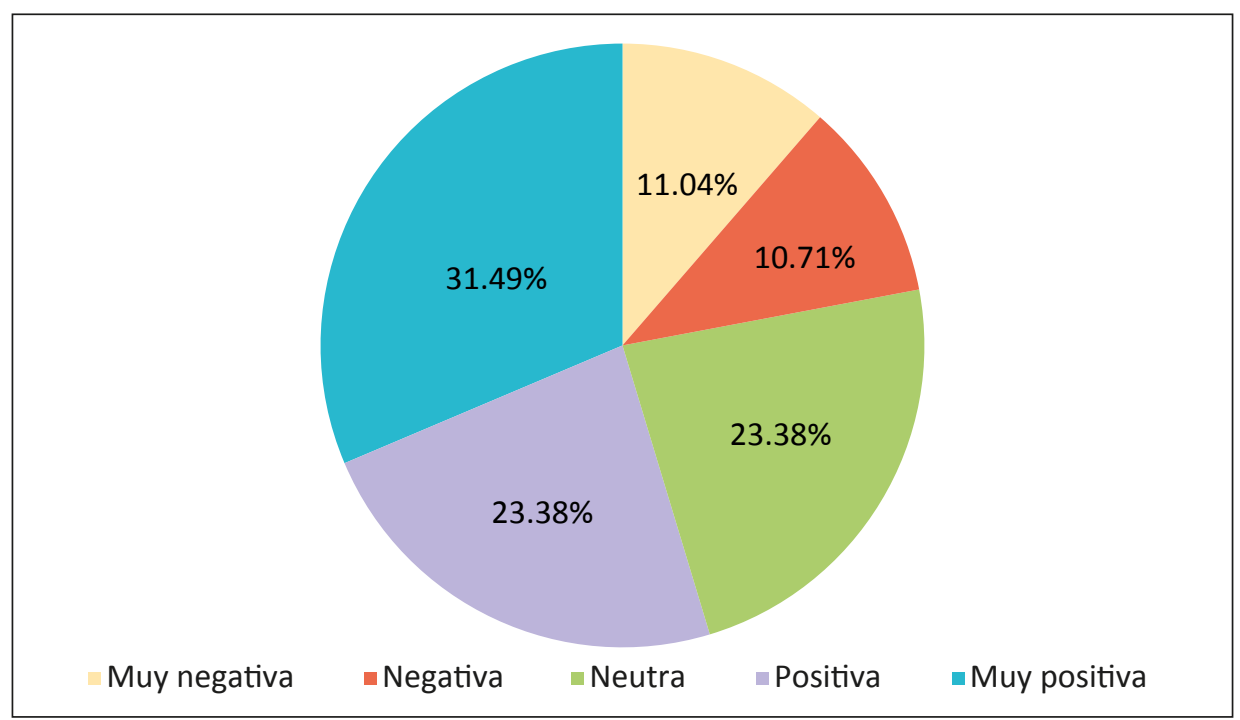

Figura 2. Distribución de resultados de las actitudes hacia los residuos sólidos. 
Respecto al objetivo específico $\mathrm{N}^{\circ} 5$, determinar la relación entre los cinco factores de personalidad y la actitud hacia los residuos sólidos según el área de residencia y en la Tabla 6 se observa que los cinco factores de la personalidad predicen la actitud positiva hacia los residuos sólidos, y adquieren especial importancia los factores de apertura, extraversión y responsabilidad.

En relación al objetivo específico $\mathrm{N}^{\circ} 6$, determinar los niveles de los factores de personalidad en la muestra, se evidencia un nivel bajo de neuroticismo, un nivel alto de extraversión, un nivel bajo de apertura a la experiencia, un nivel medio de agradabilidad y un nivel alto de responsabilidad en los participantes de la investigación (Ver Figura 1).

En lo que se refiere al objetivo específico $\mathrm{N}^{\circ} 7$, determinar la actitud hacia los residuos sólidos en la muestra, se aprecia en la Figura 2 que el mayor porcentaje de la muestra presenta actitudes favorables hacia los residuos sólidos, sin embargo se presentan también porcentajes considerables en actitudes neutras y negativas.

\section{DISCUSIÓN}

En líneas generales los hallazgos de esta investigación evidencian la utilidad del modelo de los cinco factores para comprender las diferencias individuales en la actitud hacia los residuos sólidos.

En particular, resultan ser predictores relevantes de la actitud hacia los residuos sólidos, los factores de apertura a la experiencia y responsabilidad. Los tres factores restantes presentan bajos coeficientes de correlación, y por consecuencia explican en un porcentaje menor la variación de la variable actitud.

Los resultados hallados son coherentes con los estudios previos que relacionan una mayor preocupación ambiental con mayores niveles en los rasgos de apertura a la experiencia, agradabilidad y responsabilidad (Hirsh, 2010; Hirsh \& Dolderman, 2007; Milfont \& Sibley, 2012). Seguidamente discutiremos la relación entre la actitud y cada factor de personalidad.

En relación a la apertura a la experiencia, este factor se encuentra asociado con la flexibilidad del pensamiento y a una mayor disposición o deseo de aceptar nuevas ideas éticas, sociales y políticas. Así, los sujetos abiertos tenderían a presentar una disposición favorable a las propuestas o ideas que reduzcan el impacto negativo de los residuos sólidos sobre el medio ambiente. Al respecto, se ha encontrado evidencia de que las personas relativamente liberales en sus creencias políticas, sociales y religiosas se involucran en mayor grado en la conducta de reciclar (Zapata \& Castrechini, 2011). Asimismo, la investigación conducida por Roccas, Sagiv, Schwartz y Knafo (2002) mostró que la apertura se relaciona con el valor universalismo de la autotrascendencia. Este valor caracteriza la apreciación, tolerancia y conexión espiritual y emocional con los otros y la naturaleza, en un sentimiento de formar parte de ella, derivándose en una mayor preocupación por la protección del medio ambiente (Bilsky \& Schwartz, 1994; Schultz \& Zelezny, 1999), como en los comportamientos proambientales (González, 2002). La persona abierta, de este modo, experimentaría mayor desagrado al evidenciar los efectos de la contaminación por residuos sólidos sobre los paisajes, las calles, campos, ríos, asumiendo actitudes positivas.

En contraste, los sujetos con puntuaciones bajas en apertura tienden a ser convencionales, a aceptar las costumbres e ideas que la sociedad les impone y a estar bastante acostumbrados a su manera de hacer las cosas. Roccas et.al. (2002) encontró en personas con baja apertura el valor tradición, lo que está asociado negativamente a la preocupación ambiental (Schultz \& Zelezny, 1999) y a las conductas que evidencian un consumo sano (González, 2002). Según ello, las personas cerradas son propensas a tener ideas conservadoras y materialistas en relación a la naturaleza y de los problemas que la afectan.

En lo concerniente a responsabilidad, este factor es representado por el sentido del deber, la organización, la deliberación sobre las consecuencias de las acciones y el control de los impulsos. En uno de sus sentidos, responsabilidad significa "gobernado por la conciencia", y refleja la adherencia estricta a los principios éticos propios y el cumplimiento escrupuloso de las obligaciones morales (TEA Ediciones, 2002). Algunas investigaciones han mostrado el reportar un sentido de deber por las consecuencias de la conducta en el estado del medio ambiente y en la generación de problemas ambientales se asocia a una mayor disposición a proteger el medio ambiente (Corral Verdugo, 2001; González, 2002). También, se ha encontrado este factor fuertemente correlacionado al locus de control interno (Saint-Germain,Wiernik, 
Goebel, Van Eenenburg y Klapperick, 2011), el cual se expresa en asumir responsabilidad por los problemas de contaminación que son provocados por su comportamiento, y no por culpar a terceras personas o las circunstancias. Evidencia de esto lo aporta González (2002) quien encontró que una mayor intensidad de sentimientos de culpa por arrojar basura a la calle ejerce una mayor influencia sobre conductas proambientales y del consumo sano.

Por otro lado y en contraposición, las personas con un bajo nivel de responsabilidad evidencian descuido, una actuación sin tener en cuenta las consecuencias, así como una menor rigurosidad en la aplicación de principios morales. Se puede esperar en sujetos con un nivel bajo en este factor, como señala Hirsh (2010), la búsqueda de atajos cuando se trate de realizar un comportamiento ambientalmente responsable.

Respecto al factor agradabilidad, es una dimensión de las tendencias interpersonales que se encuentra asociada al altruismo y a la empatía. Roccas, et al. (2002) hallan este factor relacionado al valor benevolencia de la autotrascendencia, que refleja una representación cognitiva del sí mismo que incluye a otras personas. Este aspecto constituye un predictor de las actitudes ambientales, de acuerdo a Berenguer (2007), al encontrar que los sujetos con un nivel alto de empatía presentaban fuertes comportamientos y actitudes ambientales. También Hirsh \& Dolderman (2007) encontraron que puntuaciones altas de agradabilidad se asocian al ecologismo.

Asimismo, Roccas, et al. encontraron la agradabilidad, asociada negativamente a los valores de autoengrandecimiento, que evidencian la valoración de metas e ideales que están orientadas hacia el beneficio propio, reflejando una definición reducida del sí mismo que no incluye a otras personas o seres vivientes (Schultz \& Zelezny, 1999). De esta forma, el consumismo que enfatiza la importancia de las posesiones materiales y la búsqueda de la riqueza personal, resultado de la contaminación por residuos sólidos, se encuentra relacionada a bajos de niveles de agradabilidad (Hirsh\& Dolderman, 2007). Por lo tanto, características de posesividad y egoísmo son incompatibles con una actitud positiva frente los problemas ambientales.

Respecto a la extraversión, este factor describe no solo la sociabilidad y preferencia por la compañía de los demás, sino que describe también la asertividad, actividad y optimismo. La cualidad activa que caracteriza a este factor ha sido relacionada a la participación en actividades voluntarias, que pueden incluir la protección y cuidado ambiental. Bekkers (2002) halló que una preferencia por la estimulación activa está relacionada positivamente con el grado de actividad de voluntariado en asociaciones sociales y recreativas. En concordancia, y con respecto a la actitud positiva hacia los residuos sólidos, estudios realizados encontraron el rasgo extraversión en las personas que participan activamente en la protección del medio (Corral Verdugo, 2001). Sin embargo, de acuerdo a Bekkers (2002) los extravertidos pueden estar involucrados en diferentes actividades voluntarias por una preferencia en "hacer y organizar cosas", independientemente del tipo de acción colectiva. Elia, Valery y de Martínez (2009) hallaron una correlación moderada entre las creencias antropocentristas y la extraversión. De este modo resulta posible suponer que las personas extrovertidas demuestren una preocupación ambiental de tipo egoísta en su búsqueda de una posición activa y de la vinculación social.

Un inesperado hallazgo en esta investigación ha sido encontrar el factor neuroticismo relacionado negativamente a la actitud hacia los residuos sólidos. La investigación realizada por Hirsh (2010) sobre la relación de este factor con el constructo general de preocupación ambiental, reportó una asociación positiva, con una mayor cantidad de individuos neuróticos demostrando significativamente altos niveles de preocupación ambiental. Hirsh (2010) ha argumentado que los individuos neuróticos tienden a estar más preocupados acerca de las consecuencias negativas en general y así demostrarían una preocupación ambiental egoísta que puede reflejar la ansiedad por las consecuencias de la degradación ambiental sobre uno mismo; mientras que individuos emocionalmente estables experimentarían potencialmente menos disturbios afectivos al pensar acerca de este tema. Sin embargo y a raíz de nuestros resultados se realiza una interpretación diferente de estos resultados. El neuroticismo describe los estados emocionales negativos, la presencia de ideas irracionales, la preocupación por aquello que puede salir mal y la pobre capacidad para enfrentarse a situaciones de estrés. Así, los resultados hallados podrían constituir un indicio, como señalan Kokkarien \& Cotgrave (2010), de que los individuos con estabilidad emocional al experimentar menor angustia son más capaces de reconocer el estado del medio ambiente que los individuos neuróticos. Más aún, el sentirse capaz de enfrentarse a los problemas de la 
contaminación, incrementarían las actitudes positivas. Para comprender la naturaleza de la relación entre el neuroticismo y las actitudes hacia el ambiente sería necesario un mayor número de investigaciones. No se rechaza la explicación de Hirsh (2010) respecto a que los individuos neuróticos también presenten una preocupación ambiental, aunque esta sea por las consecuencias que pueden resultar para uno mismo. No obstante resulta importante considerar que aunque una persona con un nivel alto de neuroticismo sienta ansiedad por los problemas ambientales, la percepción de incapacidad respecto a enfrentar estos problemas podría, no obstante, generar desesperanza y por ende actitudes negativas.

Respecto a los objetivos específicos, se procede a realizar su análisis y discusión. En relación al género, los resultados constituyen un aporte a la importancia del factor apertura a la experiencia en el establecimiento de una conexión emocional con el medio ambiente, tanto en el caso de hombres como de mujeres. Asimismo, se encontró un resultado inesperado en la relación entre agradabilidad y actitud hacia los residuos sólidos en los estudiantes universitarios hombres, con una valencia negativa. Este resultado podría estar evidenciando en este grupo, que características personales como la competitividad se relacionan a una actitud positiva, la que podría estar motivada por la obtención de cierto reconocimiento social. No obstante, la correlación encontrada presenta un coeficiente de muy baja magnitud, siendo por ende importante realizar otras investigaciones.

Respecto al período de desarrollo humano, los resultados hallados continúan corroborando las relaciones establecidas entre los factores de personalidad y la actitud aún en diferentes períodos de desarrollo.

En cuanto al ciclo académico, se encontraron diferencias en la influencia de los factores de la personalidad sobre la actitud entre los estudiantes del primer ciclo y el tercer ciclo. Estas diferencias reportaron que solo apertura a la experiencia y agradabilidad predecían la actitud positiva hacia los residuos sólidos en los estudiantes del tercer ciclo, mientras que en el caso de los estudiantes del primer ciclo, todos los factores ejercían influencia. La explicación para este hallazgo podría encontrarse en la interacción de otros factores psicológicos sobre las actitudes de los estudiantes del tercer ciclo; como pueden ser, el nivel de conocimientos y habilidades en la gestión de los residuos sólidos, los cuales podrían haberse visto incrementados a un mayor nivel de desarrollo académico.

Respecto a la carrera, se reportaron bajas correlaciones entre las variables en las carreras número uno y número dos, mientras que en la carrera número tres, las correlaciones presentaron una magnitud media y alta. Estos resultados podrían explicarse por la naturaleza de las carreras número uno y número dos, carreras que se orientan respectivamente al desarrollo y bienestar de otras personas, y la protección de otros seres vivientes, por lo cual, otros factores psicológicos podrían estar influenciando la actitud hacia los residuos.

En relación al área de residencia, los hallazgos muestran, en primer lugar, la dificultad para examinar la importancia del área de residencia como una variable moduladora al presentarse un desbalance en la proporción de participantes por área de residencia. Sin embargo y en términos generales, se evidencia que los cinco factores de la personalidad predicen la actitud positiva hacia los residuos sólidos, adquiriendo especial importancia los factores de apertura, extraversión y responsabilidad.

Sobre los niveles de los factores de personalidad, los resultados mostraron un nivel bajo de neuroticismo, nivel alto de extraversión, nivel bajo de apertura a la experiencia, nivel medio de agradabilidad y un nivel alto de responsabilidad. Asimismo, el análisis de los promedios en los cinco factores de personalidad da evidencia de un nivel medio en todos los factores. Estos resultados coinciden con los obtenidos por Contreras, Espinoza \& Esguerra (2009) y Ramírez (2007), quienes aplicaron el NEO FFI en estudiantes universitarios colombianos y peruanos, respectivamente. Esta tendencia similar en los cinco factores a pesar de las diferencias culturales constituye un apoyo en el reconocimiento de una estructura universal de la personalidad, que se encuentra constituida por cinco dimensiones fundamentales.

Los promedios similares encontrados muestran las medias más altas en los factores de responsabilidad y extraversión, mientras que neuroticismo tiene el menor valor. En relación a la responsabilidad, los resultados sugieren que los estudiantes que conforman la muestra tienden a ser organizados, responsables, concienzudos, con principios sólidos y que se esfuerzan para conseguir sus metas. Respecto a la extroversión, se halla una tendencia a ser sociables, activos y a preferir los grupos y las reuniones. En cuanto a la agradabilidad, los estudiantes tienden a ser generalmente cálidos, confiados y agradables 
con los demás, pero a veces pueden ser también testarudos y competitivos. Sobre a la apertura a la experiencia, se encontró que los estudiantes son más prácticos, convencionales, prefiriendo lo familiar a lo novedoso. En el neuroticismo, los resultados sugieren que los estudiantes tienden a ser emocionalmente estables, seguros, capaces de controlar sus impulsos y a enfrentarse de a situaciones estresantes sin alterarse ni aturdirse. En general, estos resultados pueden deberse a las exigencias académicas y otras características de la vida universitaria que fomentan la organización, actividad, competitividad, practicidad y el afrontamiento adaptativo.

En relación a las actitudes hacia los residuos sólidos en la muestra, se ha encontrado un mayor porcentaje de actitudes favorables hacia los residuos sólidos. Estos resultados coinciden con los hallazgos del Instituto de Opinión Pública (2011), que reporta que un $47.8 \%$ de los ciudadanos de Lima Metropolitana valoran la contaminación ambiental como el segundo problema más importante que afecta la calidad de vida, un $27.6 \%$ preocupado por el sistema de recojo de la basura y un $86.8 \%$ mostró una disposición a actuar para reducir la contaminación de la ciudad. Asimismo, encuestas anteriores como las del Instituto de Opinión Pública (2009) y el Grupo de Opinión Pública de la Universidad de Lima (2009) mostraron mucha preocupación por la situación del ambiente. Según lo manifestado, se demuestra una tendencia creciente de las actitudes positivas hacia el ambiente.

Sin embargo, es necesario notar también la presencia de actitudes desfavorables y neutras hacia los residuos sólidos. Una explicación para este hallazgo se podría encontrar en las dificultades y costes que implican las acciones para gestionar y reducir los residuos sólidos.

\section{CONCLUSIONES}

Los resultados de esta investigación evidencian que existe correlación entre los cinco factores de personalidad y la actitud hacia los residuos sólidos en la muestra de estudiantes universitarios. De esta forma, los resultados describen que la actitud positiva hacia los residuos sólidos está relacionada a altos niveles de apertura a la experiencia, responsabilidad, extraversión y agradabilidad, y a un nivel bajo de neuroticismo. Asimismo, dentro de estos factores, la apertura a la experiencia y la responsabilidad son principales predictores de la actitud hacia los residuos sólidos.

En relación a las variables sociodemográficas, los resultados muestran a la apertura a la experiencia como el predictor más significativo, tanto en los hombres y las mujeres. Según el período de desarrollo humano, los resultados mostraron la influencia de los cinco factores de personalidad sobre la actitud en los estudiantes universitarios que se encuentran en el período de la adolescencia y la adultez temprana. Según el ciclo académico se evidencia que en los estudiantes del primer ciclo los cinco factores de personalidad predicen en mayor medida su actitud; en cambio, en los estudiantes del tercer ciclo solo apertura a la experiencia y agradabilidad. Según la carrera, los resultados muestran que los factores de personalidad más significativos en la predicción de la actitud son agradabilidad y la responsabilidad en la Carrera número uno, apertura a la experiencia en la Carrera número dos, y extroversión, apertura y responsabilidad en la Carrera número tres. Según el área de residencia se mostró que los cinco factores de la personalidad predicen la actitud positiva hacia los residuos sólidos, adquiriendo especial importancia los factores de apertura, responsabilidad y extraversión en las diferentes áreas donde residen los sujetos estudiados.

En relación al nivel de las variables personalidad y actitud, la muestra de estudiantes universitarios presentó un nivel bajo de apertura a la experiencia, un nivel alto de responsabilidad, un nivel bajo de neuroticismo, nivel alto de extraversión y un nivel medio de agradabilidad. Asimismo, la mayoría de la muestra de estudiantes universitarios tienen actitudes positivas hacia los residuos sólidos.

\section{REFERENCIAS BIBLIOGRÁFICAS}

1. Bekkers, R. (2002). Participation in voluntary associations: resources, personality or both? Recuperado de Utrecht University, Department of Sociology: http://www.fss.uu.nl/soc/homes/bekkers/ participation.pdf

2. Berenguer, J. (2007). The effect of empathy in proenvironmental attitudes and behaviors. Environment and behavior, 39(2), 269-283.

3. Bilsky, W. \& Schwartz, S. (1994). Values and personality. European Journal of Personality, 8, 163181.

4. Contreras, F., Espinosa, J. \& Esguerra, G. (2009). Personalidad y afrontamiento en estudiantes universitarios. Universitas Psychologica, 8(2), 311322. 
5. Corral, V. (2001). Comportamiento Proambiental: Una introducción al estudio de las conductas protectoras del ambiente. Santa Cruz de Tenerife: Editorial Resma.

6. Costa, P.T., \& McCrae, R.R. (1994). Stability and change in personality from adolescence through adulthood. En C.F., Halverson, G., Kohnstamm \& R. Martin (Eds.), The developing structure of temperament and personality from infancy to adulthood (pp.139- 150). Hillsdale, NJ: LEA.

7. Elia, S., Valery, V \& De Martínez, E. (2009). Sistema de creencias ambientales en estudiantesde pregrado de la Universidad Metropolitana. Factores de personalidad, género y variablesacadémicas asociadas. Anales, 9(1), 197-226.

8. Fernández, R., Hueto, A., Rodríguez, L. \& Marcén, C. (2003). ¿Qué miden las escalas de actitudes? Análisis de un ejemplo para conocer la actitud hacia los residuos urbanos. Ecosistemas, 12(2). Recuperado de http://www.um.es/gtiweb/adrico/medioambiente/ escalas\%20actitudes.htm

9. González, A. (2002). Valores, actitudes y conductas proambientales en estudiantes peruanos. En: Corral, V. (Ed). Conductas protectoras del ambiente (165183). México: CONACYT.

10. Grupo de Opinión Pública. (2009). V Encuesta Anual sobre Medio Ambiente.Lima: Universidad de Lima. Recuperado de http://www2.ulima.edu.pe/webulima. nsf/default/F598031D89943F2F05256E630017BD4 C/\$file/barometro_social_may_2009.pdf

11. Hirsh, J. \& Dolderman, D. (2007). Personality predictors of Consumerism and Environmentalism:

A preliminary study. Personality and Individual Differences, 43 (6), 1583-1593.

12. Hirsh, J. (2010). Personality and environmental concern.Journal of Environmental Psychology, 30, 245-248.

13. Instituto de Opinión Pública (2009). Estado de la Opinión Pública: Medio ambiente. Lima: Pontificia Universidad Católica del Perú. Recuperado de http:// blog.pucp.edu.pe/media/2846/20090603-2009\%20 Medio\%20ambiente\%20-\%20Junio.pdf
14. Instituto de Opinión Pública (2011). Encuesta Lima Cómo Vamos. Lima: Pontificia Universidad Católica delPerú. Recuperado de http://www. limacomovamos. org/cm/wp-content/uploads/2012/01 / EncuestaLima ComoVamos-2011.pdf

15. Kokkarinen, N. \& Cotgrave, A. (2010). Built environment student attitudes toward the environment. Liverpool: John Moores University, School of the Built Environment. Recuperado de http://www. arcom.ac.uk/-docs/proceedings/ar2010-0173-0180_ Kokkarinen_and_Cotgrave.pdf

16. Milfont, T. \& Sibley, C. (2012). The big five personality traits and environmental engagement: Associations at the individual and societal level. Journal of Environmental Psychology, 32(2). Recuperado de http://www.sciencedirect.com/ science/article/pii/S0272494412000072

17. Ramírez, O. (2007). Propiedades psicométricas del NEO FFI (Neo Five Factor Inventory) forma $\mathrm{S}$ en un grupo de estudiantes universitarios. (Tesis inédita de Licenciatura). Universidad Peruana Cayetano Heredia, Lima, Perú.

18. Roccas, S., Sagiv, L., Schwartz, S. \& Knafo, A. (2002). The Big Five Personality Factors and Personal Values. Personality and Social Psychology Bulletin, 28(6), 789-801.

19. Saint-Germain, M.,Wiernik, B., Goebel, C., Van Eenenburg, D. \& Klapperick, J. (2011). Effect of Observed Conscientiousness on Perceptions of Locus of Control. Sentience, 4, 1-7.

20. Schultz, W \& Zelezny, L. (1999). Values as predictors of environmental attitudes: evidence for consistency across 14 countries. Journal of Environmental Psychology, 19(3), 255-265.

21. TEA Ediciones (2002). Inventario de personalidad $\mathrm{NEO}$ revisado (NEO PI-R) e Inventario NEO reducido de cinco factores (NEO-FFI). Madrid: TEA.

22. Zapata, R. \& Castrechini, A. (2011). Conducta proambiental y personalidad: Análisis de un barrio de Lima. Quaderns de Psicología, 13(1), 47-61. Recuperado de http:/www.quadernsdepsicologia.cat/ article/view/932 